ISSN 0103-9954

\title{
PRODUÇÃO DE CHAPAS DE MADEIRA COMPENSADA DE CINCO ESPÉCIES DE PINUS TROPICAIS
}

\section{PLYWOOD MANUFACTURE FROM FIVE SPECIES OF TROPICAL PINE}

\author{
Setsuo Iwakiri ${ }^{1}$ Danielle Previdi Olandoski ${ }^{2}$ Gabriela Leonhardt ${ }^{2}$ Martha Andreia Brand ${ }^{2}$
}

\section{RESUMO}

Este trabalho teve como objetivo avaliar o potencial de utilização de 5 espécies de pinus tropicais para produção de painéis compensados. As espécies estudadas foram: Pinus caribaea, Pinus chiapensis, Pinus maximinoi, Pinus oocarpa, Pinus tecunumannii e Pinus taeda, sendo esta última espécie como testemunha. Foram produzidos compensados de 5 lâminas com resinas uréia- formaldeído e fenol-formaldeído. Os resultados de inchamento e recuperação em espessura foram estatisticamente iguais entre as espécies estudadas, com exceção para inchamento em espessura das chapas coladas com resina fenol-formaldeído. As chapas de Pinus maximinoi e Pinus oocarpa, apresentaram melhores resultados de módulos de elasticidade. Para o módulo de ruptura, as chapas de Pinus maxininoi, Pinus oocarpa e Pinus taeda, coladas com resina fenol-formaldeído, apresentaram valores estatisticamente superiores em relação às demais espécies. Quanto a resistência da linha de cola, as chapas de Pinus maximinoi, Pinus taeda e Pinus chiapensis, foram as que apresentaram melhor desempenho. Com base nos resultados gerais da pesquisa, pode-se destacar a potencialidade da madeira de Pinus maximinoi e Pinus oocarpa para produção de chapas de madeira compensada.

Palavras-chave: Pinus tropicais, compensados, resistência da linha de cola.

\begin{abstract}
This research was developed aiming at evaluating the feasibility of the use of 5 species of tropical pine to plywood manufacture. The following species were studied: Pinus caribaea, Pinus chiapensis, Pinus maximinoi, Pinus oocarpa, Pinus tecunumannii and Pinus taeda, being the last used as the referential species. Plywood were manufactured with 5 plies, bonded with ureaformaldheyde and fenol-formaldheyde resin. The results of thickness sweeling and recovering were the same for all species studied, with exception to thickness sweeling for the boards glued with fenolformaldheyde resin. The boards made from Pinus maximinoi and Pinus oocarpa, showed the higher values in modulus of elasticity. The boards of Pinus maximinoi, Pinus oocarpa and Pinus taeda, glued with fenol-formaldheyde resin, resulted in higher values of the modulus of rupture, in comparison to other species. For the glue line strength, the boards of Pinus maximinoi, Pinus taeda and Pinus chiapensis, showed the better results. Based on the general results of this research it, could be said that the Pinus maximinoi and Pinus oocarpa present the high potentiality to plywood manufacture.
\end{abstract}

Key words: Tropical pine, plywood, glue line strength.

1. Engenheiro Florestal, Dr., Professor Titular do Departamento de Engenharia e Tecnologia Florestal, Setor de Ciências Agrárias, Universidade Federal de Paraná, CEP 80210-170, Curitiba (PR).

2. Engenheira Florestal, MSc., Acadêmica do Curso de Pós-Graduação em Engenharia Florestal, Setor de Ciências Agrárias, Universidade Federal de Paraná, CEP 80210-170, Curitiba (PR). 


\section{INTRODUÇÃO}

A produção de painéis compensados no Brasil é ainda a mais representativa dentre os diversos tipos de painéis reconstituídos de madeira, como aglomerado, chapas de fibras isolantes, duras e "MDF". Segundo a ABIMCI (1998), o volume produzido em 1998 atingiu $1.650 .000 \mathrm{~m}^{3}$, sendo $40 \%$ deste volume destinados para exportação e colocando o Brasil em $7^{\circ}$ lugar entre os principais países exportadores.

As espécies mais utilizadas, na produção de lâminas e compensados no Brasil, são as de folhosas provenientes da região amazônica. A partir da década de 90, passaram a ser utilizadas em quantidades expressivas madeiras de pinus provenientes de reflorestamentos, principalmente de espécies elliottii e taeda. A perspectiva é de utilização cada vez maior de madeiras de coníferas plantadas, tendo em vista boas características de laminação, e pela redução na oferta de madeiras tropicais de boa qualidade para produção de compensados. Além dos aspectos técnicos, a pressão ambiental tem contribuído para a racionalização cada vez maior do uso da floresta amazônica como fonte de matéria prima-madeira.

A obtenção de lâminas e produção de compensados de boa qualidade está estreitamente relacionada às características inerentes a madeira, ao adesivo e procedimentos empregados na colagem (MARRA, 1992).

Quanto à madeira, a espécie utilizada deve ser avaliada quanto ao seu comportamento na colagem, principalmente no que se refere a resistência da linha de cola. A densidade da madeira influencia na sua porosidade e permeabilidade do lenho. Sendo o adesivo, um líquido com determinada viscosidade, a sua formulação relacionada ao grau de extensão e a gramatura, irão influenciar na formação da linha de cola entre as lâminas (NOCK \& RICHTER, 1978). O pH e os extrativos presentes na madeira variam de acordo com as espécies e podem influenciar negativamente na cura das resinas.

As indústrias de compensados utilizam as resinas ureia-formaldeído e fenol-formaldeído, respectivamente, para produção de painéis destinados ao uso interno e externo. As classes de uso desses painéis estão relacionadas também a qualidade de lâminas utilizadas na composição do compensado (BODIG \& JAYNE, 1982). Os procedimentos empregados na colagem uréica e fenólica, variam não apenas nas formulações e gramatura, mas também no ciclo de prensagem. A pressão, tempo e temperatura de prensagem, devem ser controladas para assegurar um contato superficial adequado entre as lâminas e permitir a cura da resina. A linha de cola formada, deve ter resistência suficiente para transferência de tensões entre as lâminas durante a utilização do painel (BALDWIN, 1981; SELLERS, 1985).

Um aspecto importante relatado por TSOUMIS (1991), é com relação à largura de anéis de crescimento e alternâncias de lenhos inicial e tardio na superfície das lâminas, provenientes de espécies de rápido crescimento. As diferenças em densidade e porosidade entre essas faixas do lenho, podem interferir na formação e resistência da linha de cola entre as lâminas de um painel compensado.

Esta pesquisa foi desenvolvida com o objetivo de avaliar a potencialidade de uso de lâminas

Ciência Florestal, v. 11, n. 2, 2001 
de 5 espécies de pinus tropicais, para produção de painéis compensados com resina uréia-formaldeído e fenol-formaldeído.

\section{MATERIAIS E MÉTODOS}

As espécies utilizadas nesta pesquisa e suas respectivas massas específicas aparentes foram: Pinus caribaea $(0,435 \mathrm{~g} / \mathrm{cm})$, Pinus chiapensis $\left(0,424 \mathrm{~g} / \mathrm{cm}^{3}\right)$, Pinus maximinoi $\left(0,483 \mathrm{~g} / \mathrm{cm}^{3}\right)$, Pinus oocarpa $\left(0,505 \mathrm{~g} / \mathrm{cm}^{3}\right)$, Pinus tecunumanii $\left(0,491 \mathrm{~g} / \mathrm{cm}^{3}\right)$ e Pinus taeda $\left(0,436 \mathrm{~g} / \mathrm{cm}^{3}\right)$, sendo esta última como espécie testemunha. As árvores com idade de 10 anos, foram retiradas de plantios da empresa PISA FLORESTAL S.A., localizada no município de Ventania, Estado do Paraná. As resinas utilizadas foram a uréia-formaldeído (UF) e fenol-formaldeído (FF).

As lâminas, com $0,2 \mathrm{~cm}$ de espessura, foram seccionadas em dimensões finais dos compensados de $45 \times 45 \mathrm{~cm}$., e secadas ao teor de umidade na faixa de $10 \%$ para colagem uréica (UF) e de $6 \%$ para colagem fenólica (FF).

A formulação adotada para resina uréia-formaldeído foi a seguinte, em partes por peso: resina UF - 100, extensor - 75, água - 80, catalisador - 7. Para resina fenol-formaldeído foi: resina FF - 100, Albex - 10, extensor - 5, água - 10.

O painel foi manufaturado com 5 lâminas, tendo dimensões nominais de 45 x 45 x 1,0 cm e coladas com a gramatura de $350 \mathrm{~g} / \mathrm{m}^{2}$ (UF) e $380 \mathrm{~g} / \mathrm{m}^{2}$ (FF). O ciclo de prensagem para colagem uréica foi: temperatura $-100^{\circ} \mathrm{C}$, pressão $-10 \mathrm{~kg} / \mathrm{cm}^{2}$, tempo de prensagem -11 minutos. Para colagem fenólica foi: temperatura $-140^{\circ} \mathrm{C}$, pressão $-10 \mathrm{~kg} / \mathrm{cm}^{2}$, tempo de prensagem -9 minutos .

As variáveis de estudo foram 6 espécies e 2 tipos de resina, como demonstrado no delineamento experimental (Tabela 1) e foram produzidas 3 chapas por tratamento, num total de 36 chapas.

TABELA 1: Delineamento experimental.

\begin{tabular}{cll}
\hline Tratamento & \multicolumn{1}{c|}{ Espécie } & Resina \\
\hline 1 & Pinus caribaea & Uréia-formaldeído \\
2 & Pinus chiapensis & Uréia-formaldeído \\
3 & Pinus maximinoi & Uréia-formaldeído \\
4 & Pinus oocarpa & Uréia-formaldeído \\
5 & Pinus taeda & Uréia-formaldeído \\
6 & Pinus tecunumannii & Uréia-formaldeído \\
7 & Pinus caribaea & Fenol-formaldeído \\
8 & Pinus chiapensis & Fenol-formaldeído \\
9 & Pinus maximinoi & Fenol-formaldeído \\
10 & Pinus oocarpa & Fenol-formaldeído \\
11 & Pinus taeda & Fenol-formaldeído \\
12 & Pinus tecunumannii & Fenol-formaldeído \\
\hline
\end{tabular}

Ciência Florestal, v. 11, n. 2, 2001 
Após o condicionamento ao teor de umidade de equilíbrio de, aproximadamente, $12 \%$, os painéis foram seccionados para retirada de corpos de prova para realização de seguintes ensaios físico-mecânicos: inchamento e recuperação em espessura, resistência da linha de cola aos esforços de cisalhamento e flexão estática. Todos os ensaios foram realizados com base na norma ASTM D805 (1982). Os resultados foram avaliados pela análise de variância e teste de Tukey, ao nível de probabilidade de $95 \%$.

\section{RESULTADOS E DISCUSSÕES}

\section{Inchamento e recuperação em espessura}

Os valores médios de inchamento e recuperação em espessura são apresentados na Tabela 2.

TABELA 2: Valores médios de inchamento e recuperação em espessura.

\begin{tabular}{lc|c}
\hline \multicolumn{1}{c|}{ Tratamentos } & Inchamento (\%) & Recuperação (\%) \\
\hline Pinus caribaea $-U F$ & $5,06 \mathrm{a}$ & $2,86 \mathrm{a}$ \\
Pinus chiapensis $-U F$ & $5,49 \mathrm{a}$ & $2,89 \mathrm{a}$ \\
Pinus maximinoi $-U F$ & $5,93 \mathrm{a}$ & $2,12 \mathrm{a}$ \\
Pinus oocarpa $-U F$ & $7,09 \mathrm{a}$ & $2,60 \mathrm{a}$ \\
Pinus taeda $-U F$ & $7,03 \mathrm{a}$ & $2,80 \mathrm{a}$ \\
Pinus tecunumannii $-U F$ & $5,64 \mathrm{a}$ & $1,68 \mathrm{a}$ \\
Pinus caribaea $-F F$ & $4,85 \mathrm{a}$ & $0,79 \mathrm{a}$ \\
Pinus chiapensis $-F F$ & $5,15 \mathrm{a}$ & $0,86 \mathrm{a}$ \\
Pinus maximinoi $-F F$ & $6,56 \mathrm{~b}$ & $1,24 \mathrm{a}$ \\
Pinus oocarpa $-F F$ & $6,71 \mathrm{~b}$ & $1,58 \mathrm{a}$ \\
Pinus taeda $-F F$ & $7,75 \mathrm{c}$ & $1,30 \mathrm{a}$ \\
Pinus tecunumannii $-F F$ & $6,49 \mathrm{~b}$ & $2,17 \mathrm{a}$ \\
\hline
\end{tabular}

Em que: UF = resina uréia-formaldeído; FF = resina fenol-formaldeído; Médias seguidas por letras distintas diferm entre si ao nivel de $5 \%$ de probabilidade.

Para as chapas produzidas com resina - UF, os valores médios de inchamento em espessura variaram de 5,06\% (Pinus caribaea) a 7,09\% (Pinus oocarpa), e para recuperação em espessura, a variação foi de $1,68 \%$ (Pinus tecunumannii) a 2,89\% (Pinus chiapensis).

Tanto para inchamento, quanto para recuperação em espessura, não foram constatadas diferenças estatisticamente significativas entre as espécies estudadas. Os resultados demonstram também que, nem sempre, o maior inchamento em espessura da chapa está relacionado com a maior recuperação em espessura.

Para as chapas produzidas com resina - FF, os valores médios de inchamento em espessura variaram de 4,85\% (Pinus caribaea) a 7,75\% (Pinus taeda), e para recuperação em espessura, a variação foi de $0,79 \%$ (Pinus caribaea) a 2,17\% (Pinus tecunumannii).

As chapas de Pinus taeda apresentaram valores de inchamento em espessura estatisticamente

Ciência Florestal, v. 11, n. 2, 2001 
superiores em relação às chapas das demais espécies. Para a recuperação em espessura, não houve diferença estatística entre as espécies estudadas.

\section{Flexão estática}

Os valores médios de módulos de ruptura e de elasticidade em flexão estática são apresentados na Tabela 3 .

TABELA 3: Valores médios de módulos de ruptura (MOR) e de elasticidade (MOE).

\begin{tabular}{|c|c|c|}
\hline Tratamentos & $\operatorname{MOR}\left(\mathrm{kgf} / \mathrm{cm}^{2}\right)$ & $\operatorname{MOE}\left(\mathrm{kgf} / \mathrm{cm}^{2}\right)$ \\
\hline Pinus caribaea -UF & $348,70 \mathrm{a}$ & $38.773,43$ a \\
\hline Pinus chiapensis - UF & $382,80 \mathrm{a}$ & $42.239,14 \mathrm{a}$ \\
\hline Pinus maximinoi $-U F$ & $394,90 \mathrm{a}$ & $68.381,27 \quad b$ \\
\hline Pinus oocarpa - UF & $331,10 \mathrm{a}$ & $61.589,34 \quad b$ \\
\hline Pinus taeda $-U F$ & $320,10 \mathrm{a}$ & $43.302,70 \mathrm{a}$ \\
\hline Pinus tecunumannii - UF & $389,40 \mathrm{a}$ & $50.154,85 \mathrm{a}$ \\
\hline Pinus caribaea $-F F$ & $275,00 \mathrm{a}$ & $41.584,33 \mathrm{a}$ \\
\hline Pinus chiapensis $-F F$ & $378,40 \mathrm{a}$ & $51.274,15 \mathrm{a}$ \\
\hline Pinus maximino $-F F$ & 508,20 & $70.668,12$ \\
\hline Pinus oocarpa $-F F$ & 522,50 & $64.771,08$ \\
\hline Pinus taeda $-F F$ & 584,10 & $58.005,37 \quad b$ \\
\hline Pinus tecunumannii - FF & $431,20 \quad b$ & $46.344,30 \mathrm{a}$ \\
\hline
\end{tabular}

Em que: UF = resina uréia-formaldeído; $\mathrm{FF}$ = resina fenol-formaldeído; Médias seguidas por letras distintas diferm entre si ao nivel de 5\% de probabilidade.

Para as chapas produzidas com resina - UF, os valores médios de MOR variaram de 320,10 $\mathrm{kgf} / \mathrm{cm}^{2}$ (Pinus taeda) a 394,90 kgf/ $\mathrm{cm}^{2}$ (Pinus maximinoi), e para o MOE a variação foi de $38.773,43 \mathrm{kgf} / \mathrm{cm}^{2}$ (Pinus caribaea) a $68.381,27 \mathrm{kgf} / \mathrm{cm}^{2}$ (Pinus maximinoi).

Para o módulo de ruptura, não foram constatadas diferenças estatísticas entre as espécies estudadas. Por outro lado, as chapas de Pinus maximinoi e Pinus oocarpa apresentaram valores médios de módulo de elasticidade estatisticamente superiores em relação às demais espécies.

Para as chapas produzidas com resina - FF, os valores médios de MOR variaram de 275,00 $\mathrm{kgf} / \mathrm{cm}^{2}$ (Pinus caribaea) a $584,10 \mathrm{kgf} / \mathrm{cm}^{2}$ (Pinus taeda), e para o MOE a variação foi de $41.584,33 \mathrm{kgf} / \mathrm{cm}^{2}$ (Pinus caribaea) a 70.668, $12 \mathrm{kgf} / \mathrm{cm}^{2}$ (Pinus maximinoi).

As chapas de Pinus maximinoi, Pinus oocarpa e Pinus taeda apresentaram valores médios de MOR estatisticamente iguais entre si e superiores em relação às demais espécies estudadas. Com relação ao MOE, as chapas de Pinus maximinoi e Pinus oocarpa apresentaram valores estatisticamente superiores em relação às chapas de Pinus taeda e às demais espécies.

\section{Resistência da linha de cola ao esforço de cisalhamento}

Os valores médios de resistência da linha de cola ao esforço de cisalhamento, para testes seco, úmido e fervura, são apresentados na Tabela 4. 
TABELA 4: Resistência da linha de cola ao esforço de cisalhamento.

\begin{tabular}{l|c|c|c|c}
\hline \multicolumn{1}{c}{ Tratamentos } & $\begin{array}{c}\mathrm{UF}-\mathrm{seco} \\
\left(\mathrm{kgf} / \mathrm{cm}^{2}\right)\end{array}$ & $\begin{array}{c}\mathrm{UF}-\text { úmido } \\
\left(\mathrm{kgf} / \mathrm{cm}^{2}\right)\end{array}$ & $\begin{array}{c}\mathrm{FF}-\mathrm{seco} \\
\left(\mathrm{kgf} / \mathrm{cm}^{2}\right)\end{array}$ & $\begin{array}{c}\mathrm{FF}-\text { fervura } \\
\left(\mathrm{kgf} / \mathrm{cm}^{2}\right)\end{array}$ \\
\hline Pinus caribaea & $12,77 \mathrm{a}$ & $7,46 \mathrm{a}$ & $23,38 \mathrm{a}$ & $14,54 \mathrm{a}$ \\
Pinus chiapensis & $15,16 \mathrm{a}$ & $9,54 \mathrm{~b}$ & $30,23 \mathrm{~b}$ & $20,10 \mathrm{~b}$ \\
Pinus maximinoi & $16,21 \mathrm{a}$ & $13,17 \mathrm{~b}$ & $32,40 \mathrm{c}$ & $16,67 \mathrm{a}$ \\
Pinus oocarpa & $12,09 \mathrm{a}$ & $5,52 \mathrm{a}$ & $28,82 \mathrm{~b}$ & $13,62 \mathrm{a}$ \\
Pinus taeda & $17,79 \mathrm{a}$ & $10,65 \mathrm{~b}$ & $35,67 \mathrm{c}$ & $14,85 \mathrm{a}$ \\
Pinus tecunumannii & $13,59 \mathrm{a}$ & $6,92 \mathrm{a}$ & $25,21 \mathrm{~b}$ & $14,00 \mathrm{a}$ \\
\hline
\end{tabular}

Em que: UF = resina uréia-formaldeído; FF = resina fenol-formaldeído; Médias seguidas por letras distintas diferm entre si ao nivel de $5 \%$ de probabilidade.

Os valores médios de resistência da linha de cola para chapas produzidas com resina - UF, variaram de $12,09 \mathrm{kgf} / \mathrm{cm}^{2}$ (Pinus oocarpa) a 17,79 $\mathrm{kgf} / \mathrm{cm}^{2}$ (Pinus taeda) para teste seco, e de 5,52 $\mathrm{kgf} / \mathrm{cm}^{2}$ (Pinus oocarpa) a 13,17 kgf $/ \mathrm{cm}^{2}$ (Pinus maximinoi) para teste úmido.

Para o teste seco, não foram constatadas diferenças estatisticamente significativas entre as espécies estudadas. No teste úmido, as chapas de Pinus maximinoi, Pinus chiapensis e Pinus taeda, apresentaram médias estatisticamente iguais entre si, e superiores em relação às chapas de Pinus caribaea, Pinus oocarpa, e Pinus tecunumannii.

Os valores médios de resistência da linha de cola para chapas produzidas com resina - FF, variaram de 23,38 kgf/ $\mathrm{cm}^{2}$ (Pinus caribaea) a 35,67 kgf/ $\mathrm{cm}^{2}$ (Pinus taeda) para teste seco, e de $13,62 \mathrm{kgf} / \mathrm{cm}^{2}$ (Pinus oocarpa) a 20,10 kgf/ $\mathrm{cm}^{2}$ (Pinus chiapensis) para teste de fervura.

Para o teste seco, as chapas de Pinus maximinoi e Pinus taeda apresentaram médias estatisticamente iguais e superiores em relação às demais espécies. No teste de fervura, a média obtida para chapas de Pinus chiapensis foi estatisticamente superior em relação às demais espécies.

\section{CONCLUSÕES}

Com base nos resultados obtidos nesta pesquisa, as seguintes conclusões podem ser apresentadas:

Todas as cinco espécies de pinus tropicais estudadas, apresentaram comportamento satisfatório nos ensaios de inchamento e recuperação em espessura, inclusive em relação às chapas de Pinus taeda;

As chapas de Pinus maximinoi e Pinus oocarpa foram as que apresentaram melhores resultados para módulos de elasticidade, para o dois tipos de resina;

Os resultados de módulo de ruptura das chapas produzidas com resina uréia-formaldeído, foram estatisticamente iguais entre as espécies; Para chapas produzidas com resina fenolformaldeído, as espécies Pinus maximnoi, Pinus oocarpa e Pinus taeda apresentaram médias estatisticamente superiores em relação às demais espécies; 
De uma maneira geral, as chapas de Pinus maximinoi, Pinus taeda e Pinus chiapensis, foram as espécies que apresentaram os melhores resultados nos ensaios de resistência da linha de cola ao esforço de cisalhamento;

Com base nos resultados gerais obtidos nesta pesquisa, pode-se destacar a grande potencialidade da madeira de Pinus maximinoi e Pinus oocarpa para produção de chapas de madeira compensada.

\section{AGRADECIMENTOS}

Os autores expressam seus agradecimentos às empresas PISA FLORESTAL S.A. e ALBA QUÍMICA S.A., respectivamente, pelas doações de madeira e adesivo utilizados nesta pesquisa.

\section{REFERÊNCIAS BIBLIOGRÁFICAS}

AMERICAN SOCIETY FOR TESTING AND MATERIALS. ASTM D-805-63: Standard methods of testing veneer, plywood, and other glued veneer constructions. Philladelphia, 1971.

ASSOCIAÇÃO BRASILEIRA DA INDÚSTRIA DE MADEIRA COMPENSADA E INDUSTRIALIZADA. Relatório estatístico. São Paulo, 1998.

BALDWIN, R.F. Plywood manufacturing practices. San Franacisco: Miller Freeman 1981. 326p.

BODIG, J.; JAYNE, B.A. Mechanins of wood and wood composites. New York: Van Nostrand Reinhold, 1982. 711p.

MARRA, A.A. Technology of wood bonding: principle in practice. New York: Van Nostrand Reinhold, 1992. 453p.

NOCK, H.P.; RICHTER, H.G. Adesão e adesivos. Curitiba: Universidade Federal do Paraná, 1978. 93p.

SELLERS JUNIOR, T. Plywood and adhesive technology. New York: Marcel Dekker, Inc, 1985. 661p.

TSOUMIS, G. Science and technology of wood: structure, properties and utilization. New York: Chapman \& Hall, 1991. 494p. 